\title{
Una aproximación a la prospección arqueológica desde la legislación y la perspectiva administrativa
}

\section{An approach to the archaeological prospection under the legislative and administrative perspective}

ANA MATEOS OROZCO

ORCID: https://orcid.org/0000-0002-8778-5116

Universidad de Sevilla. Departamento

de Prehistoria y Arqueología

amorozco@us.es

Fecha de recepción: 29-05-2019

Fecha de aceptación: 15-06-2019

DOI: http://dx.doi.org/10.12795/9788447221912.017

Pp.: 402-423 


\section{Resumen}

La innovación docente está cobrando un enorme protagonismo en la actualidad. Su método y actividades proporcionan al docente herramientas y modos de ver la docencia que permiten la obtención de mejores resultados en ambas direcciones, tanto para el alumnado como para el profesor. En nuestro caso, probamos la aplicación de los denominados Ciclos de Mejora en el Aula (CIMA) a un bloque temático complejo y hostil para el alumnado, la Legislación sobre Patrimonio Histórico, y más concretamente la relación de esta con la prospección arqueológica, su labor administrativa, los aportes del arqueólogo a la protección patrimonial y las infracciones y sanciones más comunes al patrimonio arqueológico, previsibles en muchos casos. Con este ciclo de mejora, buscamos acercar esta temática al alumnado, hacerla sencilla y comprensible y, sobre todo, generar un vínculo entre el alumno y la legislación, una herramienta fundamental para el desarrollo de la práctica profesional del arqueólogo.

Palabras clave: La Prospección Arqueológica; Grado en Arqueología; Docencia universitaria; Experimentación docente universitaria; Patrimonio arqueológico.

\section{Abstract}

Educational Innovation plays a main role in the current university education. The methods and activities suggested are useful and beneficial tools both for students and teachers. In our case we tested the application of the so-called Improvement Cycles in Classroom (ICIC) to a difficult and unfriendly topic for students, Historical Heritage Laws and its relations with archaeology, archaeological prospection, its administrative task and how archaeologist can be essential preventing damages in archaeological heritage. We've tried to make this topic attractive to students and, furthermore, to make it simple and understandable and, above all, to generate a link between the student and the legislation, a fundamental tool for the development of the archaeologist's professional practice.

Keywords: Archaeological Prospection; Degree in Archaeology; University teaching; University teaching experimentation; Archaeological heritage.

Jornadas de Formación e Innovación Docente del Profesorado | № 2 (2019) Esta obra se distribuye con la licencia Creative Commons 


\section{Descripción breve del contexto}

La asignatura "La Prospección Arqueológica" es una materia obligatoria inserta en el segundo cuatrimestre del segundo curso del Grado en Arqueología. Forma parte de las denominadas metodologías básicas de intervención e investigación arqueológica, junto con la Excavación Arqueológica, Análisis de la Cultura Material, El Registro Arqueológico y Teoría e Historia de la Arqueología. Como podemos observar, es parte de un conglomerado de asignaturas que se complementan para dar lugar a lo que podríamos denominar formación esencial metodológica del arqueólogo.

La asignatura tiene una duración de 6 créditos de clases teórico-prácticas, en los cuales el docente debe proporcionar la información y formación necesaria, tal y como los conceptos básicos de prospección arqueológica, su funcionalidad, metodologías y técnicas relacionadas, casos de estudio, etc. El bloque final de la asignatura, "Interpretación, sociedades y territorio", contiene un subapartado dedicado a la práctica profesional de la prospección, en el cual se trata su dimensión como labor administrativa y labor investigadora. En este punto consideramos necesario hacer hincapié en la dimensión legislativa de la prospección, teniendo en cuenta tanto los condicionantes que encontramos en la legislación para su práctica como los aportes que proporciona la prospección arqueológica en el planeamiento territorial y la protección del patrimonio.

Es aquí donde nos planteamos aplicar el ciclo de mejora al apartado de "Legislación", por diversos motivos. Principalmente porque consideramos que la legislación es, en primera instancia, mucho más compleja que otros bloques de contenido; por otro lado, porque es un elemento fundamental en la práctica laboral del arqueólogo y su comprensión es, asimismo, fundamental para un correcto desarrollo de la profesión; finalmente por facilitar el trabajo del docente intentando hacer más ameno un temario que aparentemente no es interesante para el alumnado. 


\section{Diseño previo del Ciclo de Mejora en el Aula}

\section{Objetivos y contenidos}

Los objetivos que nos propusimos lograr durante este Ciclo de Mejora en el Aula (CIMA) fueron los siguientes:

- Facilitar la comprensión de la legislación por parte del alumnado, incorporando ayuda extra para entender los textos legislativos, repasando las leyes, creando esquemas y resúmenes y priorizando los aspectos más relevantes.

- Hacer de una temática aparentemente dificil y aburrida un elemento de interés y utilidad para la práctica laboral. En este sentido entendemos que, a pesar de que la temática no es la favorita a primera vista, podemos hacer entender al alumnado que la necesitarán en su vida profesional, que se pueden beneficiar de la misma y que es una herramienta de gran importancia.

- Acercar al alumnado a la realidad de la práctica profesional. Esto está muy en relación con el punto anterior, ya que permitirá al alumnado conocer la realidad de la profesión, con todos los pros y contras, la burocracia, el sentido administrativo, etc. Conocer este aspecto "real" puede animarlos a que presten más interés, puesto que es algo que les servirá en un futuro.

- Crear conciencia patrimonial y social. El alumnado debe ser consciente de la importancia de su trabajo y su labor con la sociedad. A menudo tendemos a pensar que las aportaciones de las carreras de humanidades son menos importantes o relevantes para el mundo actual, pero no es así. La labor del arqueólogo, historiador, etc. ayuda en muchos sectores de la población: turismo, identidad, políticas sociales, desarrollo sostenible, etc. 
- Generar empatía hacia el patrimonio (en concreto arqueológico) por parte de los alumnos. El futuro trabajador en temas patrimoniales no puede ser ajeno o apático ante el patrimonio, en primer lugar, porque trabaja con bienes delicados y de gran importancia para una comunidad, y en segundo, porque debemos dejar claro que hay que actuar conforme a la ley, poniendo el patrimonio y su defensa en primer lugar.

Estos objetivos implican toda una serie de contenidos conceptuales, procedimentales y actitudinales que se relacionan de manera directa, como queda reflejado en el siguiente cuadro (Tabla 1). Los objetivos, como podemos ver, son transversales a todos los contenidos.

Tabla 1. Objetivos y contenidos

\begin{tabular}{|c|c|c|}
\hline $\begin{array}{l}\text { Tipo de } \\
\text { contenido }\end{array}$ & Contenido & Objetivos para lograr \\
\hline \multirow{4}{*}{ CONCEPTUALES } & $\begin{array}{l}\text { El concepto de Ley de Patrimonio } \\
\text { Histórico. }\end{array}$ & \multirow{2}{*}{$\begin{array}{l}\text { Facilitar la comprensión } \\
\text { de la legislación.. }\end{array}$} \\
\hline & \multirow{2}{*}{$\begin{array}{l}\text { Definición de Patrimonio Arqueoló- } \\
\text { gico, Prospección, Catálogo, Inventa- } \\
\text { rio, BIC. }\end{array}$} & \\
\hline & & \multirow{2}{*}{$\begin{array}{l}\text { Hacer de ella un ele- } \\
\text { mento de interés y } \\
\text { utilidad }\end{array}$} \\
\hline & $\begin{array}{l}\text { Herramientas de protección, fun- } \\
\text { cionalidad y fuerza de protección. }\end{array}$ & \\
\hline \multirow{4}{*}{ PROCEDIMENTALES } & $\begin{array}{l}\text { Búsqueda y sistematización de la } \\
\text { información. }\end{array}$ & \multirow{4}{*}{$\begin{array}{l}\text { Acercar al alumnado a la } \\
\text { realidad de la práctica } \\
\text { profesional. }\end{array}$} \\
\hline & Investigación. & \\
\hline & $\begin{array}{l}\text { Trabajo, búsqueda y manejo de Car- } \\
\text { tas Arqueológicas, Catálogos y Planes } \\
\text { de Ordenación. }\end{array}$ & \\
\hline & Dominio de la LPHE y LPHA. & \\
\hline
\end{tabular}

Jornadas de Formación e Innovación Docente del Profesorado | № 2 (2019) Esta obra se distribuye con la licencia Creative Commons Reconocimiento-NoComercial-SinObraDerivada Internacional (CC BY-NC-ND 4.0.) 
Estableciendo objetivos y contenidos, generamos una pregunta clave estructuradora del contenido: ¿Qué función tiene la práctica profesional de la prospección arqueológica? Podemos realizar prospecciones en el marco de la investigación académica o en el marco administrativo, pero este segundo marco es el que más nos interesa para nuestro bloque temático, puesto que el marco administrativo supone la creación de catálogos, inventarios y Cartas Arqueológicas que sirven de base para el planeamiento territorial y la protección específica de yacimientos, áreas y entornos. A partir de esta pregunta clave, los contenidos se desarrollan de manera casi directa, estando todos relacionados con ella. Estos contenidos se trabajan a modo de problemas a resolver, algo que podemos ver en el mapa esbozado mediante diferentes cuestiones (Figura 1).

Desde estas cuestiones abordamos también la profundidad de las leyes de patrimonio (tanto la española como las de las comunidades autónomas), su eficacia, principales sanciones y fuerza a la hora de aplicarlas. Para acompañar esta parte veremos las principales infracciones en patrimonio arqueológico: destrucción, abandono y expolio, destacando la importancia de la prospección para prevenir estas acciones. Este boque temático busca despertar en el alumnado el interés por la legislación y el patrimonio arqueológico, generando debate, espíritu crítico y fomentando la investigación y el trabajo en grupo para reflexionar sobre la labor del arqueólogo en la protección del patrimonio y su labor con la sociedad. 
En el mapa de contenidos y problemas (Figura 1) se muestran los contenidos ya mencionados del bloque temático y sus problemas detectados a modo de cuestiones. Los contenidos se acompañan de herramientas o claves como las cartas arqueológicas, la legislación existente y otros documentos que facilitan la compresión de los diferentes conceptos, el poder ampliar la información y trabajar con estos documentos de forma ágil. Estas herramientas o ideas son el paso intermedio entre los conceptos y las preguntas a resolver, actuando como puente entre ambos. 


\section{ANA MATEOS OROZCO}

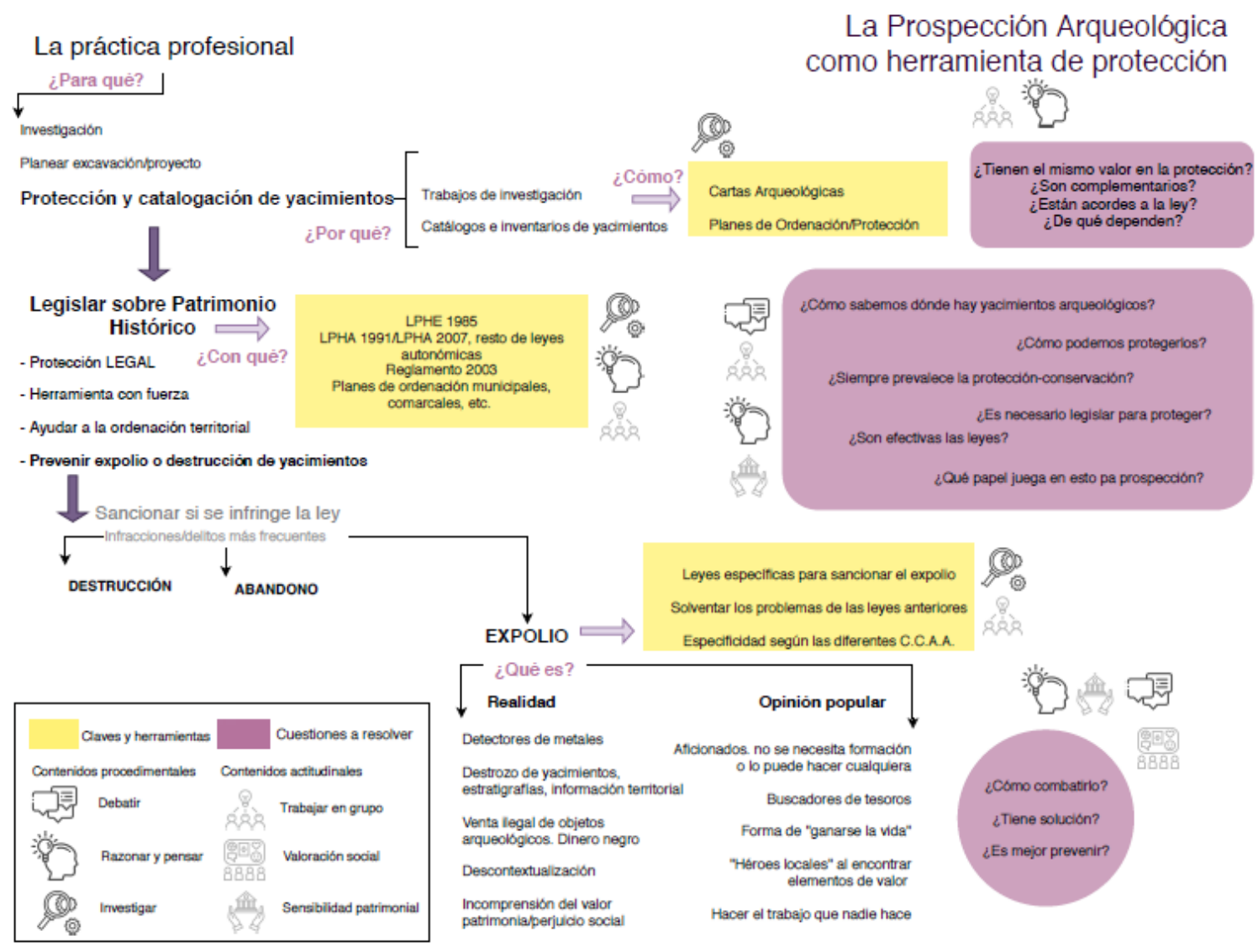

Figura 1. Mapa de contenidos y problemas.

Jornadas de Formación e Innovación Docente del Profesorado I № 2 (2019) 


\section{Modelo metodológico y secuencia de actividades}

Para lograr los objetivos planteados consideramos necesario usar como modelo metodológico docente el modelo alternativo o constructivista, por varias razones. En primer lugar, entendemos que, si tenemos en cuenta que el aprendizaje es un proceso constructivo y reestructurante, debemos orientar hacia esto nuestra forma de enseñar. Consideramos, por tanto, que debemos basar las clases en una serie de preguntas clave -como se ha reflejado en el mapa de contenidos y problemas- que permitan, en primer lugar, poner en juego los conceptos más importantes y, en segundo lugar, que los alumnos consigan un aprendizaje realmente significativo (De Alba y Porlán, 2017). Para que estas preguntas y conceptos tengan la fuerza estructurante que necesitamos, es obligado tener en cuenta las ideas iniciales del alumnado (García Pérez, 2000; Rivero y Porlán, 2017).

Esta metodología de enseñanza que hemos tomado concibe el aprendizaje como un proceso de resolución e investigación de problemas a partir y desde el diseño y el desarrollo de diversas actividades, encadenadas y orientadas a la construcción progresiva del conocimiento, teniendo como referencia un modelo didáctico centrado en el alumno (García Pérez y Porlán, 2017).

Estos problemas, que finalmente desembocan en ideas o contenidos se trabajan en diferentes tipos de actividades: clases expositivas, debates y "talleres conceptuales" (Finkel, 2008). Estos últimos suponen necesariamente la participación activa del alumnado, que construye su propio conocimiento a partir de distintas actividades.

Propusimos por tanto la siguiente secuencia de actividades:

1. Cuestionario inicial de ideas iniciales acompañado de una noticia de prensa. La noticia servía como 
llamada de atención ante la vulnerabilidad del patrimonio y la facilidad del expolio, un problema al que socialmente no se le da importancia y tiende a normalizarse.

2. Debate inicial, comentario de la noticia y tormenta de ideas sobre legislación, patrimonio histórico y la labor del arqueólogo y la prospección arqueológica. Este debate pretende ser el punto de inflexión desde el cual desarrollar el resto de actividades, ya que con él se podrá ver desde qué punto exacto parten los alumnos en la mayoría de conceptos que pretendemos tratar.

3. Exposición de las leyes existentes y herramientas de protección del patrimonio. Esta actividad consiste en exponer al alumnado la legislación sin entrar a fondo en su explicación. Además, se aportan y muestran las herramientas de protección más comunes, como Cartas Arqueológicas, Planes de Ordenación y Protección, etc. El objetivo principal en este caso es enseñar al alumnado a trabajar con estos documentos, saber dónde encontrarlos y qué hacer con ellos.

4. Creación de esquemas: La prospección arqueológica y la LPHA 2007. En este caso sí se trabaja a fondo la legislación por parte de los estudiantes, quienes deberán desglosarla para obtener todos aquellos datos relevantes y relacionados con la prospección arqueológica.

5. Resolución de casos legales sobre patrimonio. Esta actividad intenta poner a prueba las dos anteriores, ya que los alumnos deben resolver casos reales usando el material trabajado previamente. No importa que la resolución no sea del todo correcta, sino que sepan dónde pueden obtener la información, que argumenten con conocimiento y sobre todo que conozcan la realidad legal del patrimonio. 
6. Puesta en común final y repaso. Esta actividad simplemente consiste en resumir en un esquema realizado en común el contenido de la legislación sobre patrimonio en relación a la prospección arqueológica y la labor que tiene esta para la protección administrativa del patrimonio arqueológico.

7. Evaluación final. Cuestionario final y evaluación del aprendizaje. Finalmente se pasará de nuevo el cuestionario pasado, para poder evaluar, al término de la experiencia, la evolución en el aprendizaje del alumnado.

\section{Cuestionario de exploración de ideas del alumnado}

En el cuestionario inicial recogíamos varias cuestiones relacionadas con el patrimonio y su legislación, así como sobre expolio y conciencia patrimonial. El cuestionario iba acompañado de una noticia de prensa en cierto modo polémica, en ella se alababa la actuación de una persona que con un detector de metales buscaba objetos arqueológicos, algo que es ilegal en toda España. La noticia buscaba crear cierta confusión en el alumnado y hacerle reflexionar sobre el expolio, asumido y generalizado hasta tal punto que es algo muy peligroso.

Las preguntas del cuestionario eran las siguientes:

1. ¿Es legal usar detector de metales sin una autorización previa? ¿Por qué?

2. ¿Es esto igual en Andalucía, Galicia, Extremadura o cualquier otra comunidad?

3. ¿Sabes qué significan las siglas LPHE/LPHA?

4. ¿Crees que es útil la legislación en materia de patrimonio?

5. ¿Por qué se expolia el patrimonio histórico? ¿Quién lo compra? 
6. ¿Qué medios tenemos para prevenir esto? ¿Cuál es la labor del arqueólogo? (Especialmente la prospección).

El resultado de los cuestionarios iniciales nos sirvió para dos cosas fundamentales en la innovación docente, por un lado, para conocer el estado previo de los alumnos ante la temática y poder ajustar el nivel de los contenidos y orientar las actividades de una u otra forma; por otro, para que en un futuro pudiésemos evaluar la evolución del alumnado y el grado de mejora en su aprendizaje, una vez que se pasara el cuestionario en el momento final del ciclo. Como vemos, estos cuestionarios son una herramienta de enorme utilidad, que combinado con las denominadas "escaleras de aprendizaje" nos permite valorar de manera efectiva y objetiva el proceso de aprendizaje (Rivero y Porlán, 2017).

Este cuestionario se completó con una actividad de debate, también orientada a comentar la noticia sobre la que versaba, en el que los alumnos pudieron mostrar sus inquietudes, intereses y dificultades acerca del tema. Así pudimos tomar una referencia desde la que partir para comenzar las sesiones y, además, saber sobre qué elementos podíamos incidir para llamar más la atención del alumnado, generar alguna emoción en los mismos u opiniones.

\section{Principios didácticos que han guiado la experiencia}

Los principios didácticos que han guiado mi experiencia se basan principalmente en los propuestos por Ken Bain en su libro Lo que hacen los mejores profesores universitarios (2006), aunque hemos realizado varios añadidos y, en algún caso, leves modificaciones. 
En primer lugar, y como punto de partida, consideramos necesario fomentar un entorno para el aprendizaje y el pensamiento crítico, apostar por el pensamiento, el cuestionar las ideas, el debate. Esto se puede lograr generando preguntas de impacto, problemas generales o generando cuestiones a resolver por el propio alumnado con el objetivo de favorecer e incentivar su inquietud investigadora (Bain, 2006; Gureckis y Markant, 2012).

Muy en relación con lo anterior, apostamos por tener en cuenta las ideas iniciales del alumnado, o, como señala Bain, comenzar con los estudiantes en lugar de con la disciplina. Así desde un primer momento seremos conscientes de cómo actúa el alumno, qué ideas y paradigmas preconcebidos traen y qué queremos y podemos intentar corregir (Bain, 2006).

Para abordar las ideas previas del alumnado, ponerlas en cuestión e intentar cambiarlas, es necesario que tengamos muy claro qué contenidos queremos enseñar y en qué nivel de formulación. La propuesta de contenidos es el resultado de la creación de esquemas y de la sistematización, seleccionando de esta manera los que consideremos más importantes, relevantes y estructurantes. Con ello pretendemos conseguir que el alumnado interiorice al menos aquello que sabemos que es de vital importancia, intentando formularlo en un nivel que conecte con las ideas iniciales del alumnado. En este punto son fundamentales los esquemas o mapas de contenidos, ya que nos permiten ver de manera clara cuáles son esos que hemos denominado contenidos necesarios (García Díaz, Porlán y Navarro, 2017). Basándonos en la idea de Cowan (2001) que afirma que solo podemos asimilar hasta un máximo de cuatro ideas por cada lección, exposición, etc., diseñamos las actividades de las sesiones de manera que no se supere este número.

Jornadas de Formación e Innovación Docente del Profesorado | № 2 (2019) Esta obra se distribuye con la licencia Creative Commons 
En este contexto el profesor debe permitir y favorecer que el alumnado cree su propio aprendizaje y construya su conocimiento de manera autónoma. En este sentido, el alumno es activo y protagonista y el docente actúa como guía (Guerra Martín, Lima y Lima, 2017).

Finalmente, aunque no menos importante, las experiencias de aprendizaje creadas por el docente deben ser preferentemente diversas, esto estimula el cerebro, favorece la atención del alumnado y genera que el aprendizaje se dé tanto de manera secuencial como global, tal y como indicaba Norden: "the brain loves diversity" ("el cerebro adora la diversidad"). No hay un estilo exclusivo, hay una enorme variedad de la que podemos sacar enormes beneficios (Bain, 2006).

\section{Aplicación del Ciclo de Mejora en el Aula}

El ciclo de mejora se desarrolló en 8 horas, en las cuales tuvieron lugar las actividades que previamente mencionamos. En casi todas estas actividades tuvimos como núcleo central una batería de preguntas a las que dar respuesta. A continuación, mostraremos brevemente los resultados y el desarrollo de cada una de estas actividades.

1. Cuestionario inicial de ideas previas acompañado de una noticia de prensa.

En este primer cuestionario participaron más alumnos que en el final. Los resultados -como ya dijimos- permitieron seleccionar los contenidos de más relevancia o que generaban más dudas entre el alumnado. La polémica noticia de la que se acompañaba provocó las respuestas que buscábamos, ya que muchos de ellos reaccionaron ante esta de forma negativa. Si bien es cierto que algunos contestaron varias preguntas basándose en la noticia, señalando que, pese a que no pensaban eso, habían cambiado de parecer tras la lectura de la misma. 
2. Debate inicial, comentario de la noticia y tormenta de ideas sobre legislación, patrimonio histórico y la labor del arqueólogo y la prospección arqueológica.

De manera inmediatamente posterior al cuestionario, realizamos un debate a modo de asamblea donde los alumnos pudieron expresar sus dudas principales e intereses, sirviendo esto como apoyo para el desarrollo del bloque temático. Los alumnos mostraron mucho interés, especialmente después de la polémica noticia expuesta en los cuestionarios. A pesar de que tomó más tiempo del previsto en un primer momento, fue clave para la estructuración de las sesiones posteriores, ya que pudimos ver que la mayoría de los alumnos contaban con los conocimientos básicos sobre patrimonio, y que todos ellos estaban firmemente concienciados en relación con la importancia de proteger el patrimonio arqueológico y su vulnerabilidad.

3. Exposición de las leyes existentes y herramientas de protección del patrimonio.

Una vez tuvimos claro qué conceptos debíamos afianzar, procedimos a la exposición del material básico en materia de legislación. Expusimos por tanto la Ley de Patrimonio Histórico Español de 1985 y la Ley de Patrimonio Histórico Andaluz de 2007, así como el Reglamento de Actividades Arqueológicas de 2003. Acompañando a estos documentos mostramos herramientas de utilidad como las Cartas Arqueológicas, Planes de Ordenación Urbana o Planes de Protección Especial. Esta sesión era sin duda mucho más compleja de abordar, pero se buscó facilitar la interacción entre los alumnos y el profesor, minimizando el margen de dudas. Para el debate utilizamos básicamente estas cuestiones:

- ¿Consideras que las leyes existentes son útiles?

- ¿Qué herramienta de protección/ordenación es más eficaz?

- ¿Cómo puede contribuir a la creación de estas herramientas el arqueólogo? 
4. Creación de esquemas: La prospección arqueológica y la LPHA 2007.

En esta actividad los alumnos debían realizar esquemas usando el material expuesto en la anterior, principalmente la LPHA 2007; estos debían recoger aquellos conceptos más relevantes e importantes en relación a la prospección arqueológica, el desarrollo profesional de la misma y su labor como herramienta administrativa y de protección. Estos esquemas no salieron como habíamos previsto, y decidimos repetirlos en común, para lograr que fueran más efectivos. Este segundo desarrollo de la actividad tuvo lugar en la actividad 6 (puesta en común final y repaso). Se utilizaron las siguientes cuestiones:

- ¿Qué es la prospección arqueológica? ¿Para qué se usa?

- ¿En qué sentido está esto relacionado con la legislación sobre patrimonio?

- ¿Podemos prevenir el expolio y la destrucción del patrimonio arqueológico?

- ¿Cómo se legisla en la LPHA sobre la prospección arqueológica?

- ¿Cuál es la labor del prospector/arqueólogo para la administración?

- ¿Somos útiles en la lucha contra la destrucción de patrimonio arqueológico?

5. Resolución de casos legales sobre patrimonio.

Esta actividad parecía la más complicada a simple vista. En la base del Centro de Documentación Judicial (http:/ / www.poderjudicial.es) buscamos resoluciones de casos relacionados con patrimonio arqueológico, los cuales abordaban los conceptos principales del bloque temático: la labor de la prospección en la administración y protección y el expolio y destrucción de patrimonio. Para esta actividad dividimos la clase en grupos que iban recibiendo pistas conforme avanzaba la clase. Seguimos el modelo de los "talleres conceptuales" (Finkel, 2008) y de aprendizaje por construcción, ya que todas las conclusiones a las 
que llegaran debían ir acompañadas por un argumento de peso, en este caso artículos concretos de la legislación o casos y sentencias precedentes. Las cuestiones que guiaron el debate fueron estas:

- ¿Consideras que en estos casos la ley se ha aplicado justamente?

- Teniendo esto en cuenta, ¿es práctica y útil la Ley de Patrimonio?

- ¿Qué papel jugamos en esto los arqueólogos?

6. Puesta en común final y repaso.

Esta actividad estaba enfocada como un repaso final o general del bloque temático; con ella lograríamos afianzar los conceptos más complejos, resolver dudas finales y sobre todo crear un esquema que mejorara los anteriores y que los alumnos pudieran usar como herramienta fundamental para el estudio del bloque. Usando la pizarra como eje central de la clase, expusimos en esta todas las ideas surgidas de los esquemas anteriores, y seleccionamos solo aquellas verdaderamente necesarias y relevantes para su desarrollo. Creamos el esqueleto del esquema, el cual debían completar los alumnos en casa. Estos segundos esquemas fueron sin duda mejores que los primeros, ya que además de la ayuda en clase los alumnos se ciñeron a dar respuesta a las preguntas propuestas.

7. Evaluación final. Cuestionarios finales y evaluación del aprendizaje.

Finalmente se pasó el cuestionario final (debemos indicar aquí que la participación del alumnado fue mucho menor por diversas cuestiones: carácter voluntario de los cuestionarios y prácticas de campo de otras asignaturas). Solo contamos con 13 cuestionarios, de manera que, aunque la muestra fuera menor en tamaño, pudimos realizar un análisis mucho más intenso, como se expondrá más adelante. En este caso pudimos comprobar que todos los alumnos mejoraron de manera significativa. 


\section{Evaluación del aprendizaje del alumnado}

Comparando los cuestionarios iniciales y finales pudimos observar el cambio y evolución en el aprendizaje del alumnado. Se estipularon 4 niveles con el objeto de valorar este aprendizaje, distinguiendo los diferentes niveles con números y colores: 0-blanco: no sabe la respuesta; 1-amarillo: sabe algo, pero muy vagamente; 2-rojo: sabe la respuesta, pero no la explica con total claridad; 3-verde: sabe la respuesta y la argumenta. Este sistema se aplicó en ambos casos, creando finalmente un cuadro comparativo en el que pudimos verlos de manera simultánea. Añadimos además una tercera columna en la que mediante flechas o signos de igual indicábamos si el alumno había mejorado su respuesta en uno, dos o hasta tres niveles, había bajado o se había quedado en el mismo nivel (Figura 2).

\begin{tabular}{|c|c|c|c|c|c|c|c|c|c|c|c|c|c|c|c|c|c|c|}
\hline $\begin{array}{c}\text { CUESTIONARIOS } \\
\text { PATRIMONIO }\end{array}$ & \multicolumn{2}{|c|}{ Cuestión 1} & \multicolumn{3}{|c|}{ Cuestión 2} & \multicolumn{3}{|c|}{ Cuestión 3} & \multicolumn{3}{|c|}{ Cuestión 4} & \multicolumn{3}{|c|}{ Cuestión 5} & \multicolumn{3}{|c|}{ Cuestión 6} \\
\hline ALUMNO 1 & 1 & 2 & $\uparrow$ & 0 & 2 & $\uparrow \uparrow$ & 0 & 3 & $\uparrow \uparrow \uparrow$ & 1 & 3 & $\uparrow \uparrow$ & 2 & 3 & $\uparrow$ & 2 & 3 & $\uparrow$ \\
\hline ALUMNO 2 & 1 & 3 & $\uparrow \uparrow$ & 2 & 2 & $=$ & 1 & 3 & $\uparrow \uparrow$ & 2 & 3 & $\uparrow$ & 2 & 3 & $\uparrow$ & 2 & 3 & $\uparrow$ \\
\hline ALUMNO 3 & 2 & 2 & $=$ & 3 & 3 & $=$ & 2 & 3 & $\uparrow$ & 2 & 2 & $=$ & 2 & 3 & $\uparrow$ & 1 & 2 & $\uparrow$ \\
\hline ALUMNO 4 & 2 & 3 & $\uparrow$ & 0 & 0 & $=$ & 0 & 2 & $\uparrow \uparrow$ & 1 & 1 & $=$ & 1 & 2 & $\uparrow$ & 2 & 2 & $=$ \\
\hline ALUMNO 5 & 0 & 3 & $\uparrow \uparrow \uparrow$ & 0 & 1 & $\uparrow$ & 0 & 3 & $\uparrow \uparrow \uparrow$ & 0 & 2 & $\uparrow \uparrow$ & 2 & 2 & $=$ & 1 & 2 & $\uparrow$ \\
\hline ALUMNO 6 & 0 & 3 & $\uparrow \uparrow \uparrow$ & 1 & 3 & $\uparrow \uparrow$ & 1 & 3 & $\uparrow \uparrow$ & 1 & 2 & $\uparrow$ & 2 & 3 & $\uparrow$ & 2 & 3 & $\uparrow$ \\
\hline ALUMNO 7 & 2 & 3 & $\uparrow$ & 2 & 2 & $=$ & 0 & 3 & $\uparrow \uparrow \uparrow$ & 1 & 2 & $\uparrow$ & 1 & 2 & $\uparrow$ & 1 & 2 & $\uparrow$ \\
\hline ALUMNO 8 & 2 & 2 & $=$ & 1 & 2 & $\uparrow$ & 0 & 3 & $\uparrow \uparrow \uparrow$ & 2 & 2 & $=$ & 2 & 2 & $=$ & 1 & 3 & $\uparrow \uparrow$ \\
\hline ALUMNO 9 & 1 & 2 & $\uparrow$ & 0 & 3 & $\uparrow \uparrow \uparrow$ & 1 & 3 & $\uparrow \uparrow$ & 2 & 3 & $\uparrow$ & 2 & 2 & $=$ & 1 & 1 & $=$ \\
\hline ALUMNO 10 & 0 & 3 & $\uparrow \uparrow \uparrow$ & 0 & 2 & $\uparrow \uparrow$ & 0 & 2 & $\uparrow \uparrow$ & 3 & 3 & $=$ & 2 & 3 & $\uparrow$ & 1 & 3 & $\uparrow \uparrow$ \\
\hline ALUMNO 11 & 1 & 1 & $=$ & 2 & 2 & $=$ & 2 & 3 & $\uparrow$ & 2 & 3 & $\uparrow$ & 2 & 3 & $\uparrow$ & 2 & 2 & $=$ \\
\hline ALUMNO 12 & 1 & 2 & $\uparrow$ & 0 & 3 & $\uparrow \uparrow \uparrow$ & 0 & 3 & $\uparrow \uparrow \uparrow$ & 1 & 2 & $\uparrow$ & 3 & 2 & $\downarrow$ & 1 & 2 & $\uparrow$ \\
\hline ALUMNO 13 & 1 & 3 & $\uparrow \uparrow$ & 2 & 3 & $\uparrow$ & 0 & 3 & $\uparrow \uparrow \uparrow$ & 1 & 2 & $\uparrow$ & 1 & 2 & $\uparrow$ & 1 & 1 & $=$ \\
\hline
\end{tabular}

Figura 2. Resumen de la evolución individual del aprendizaje del alumnado.

El resultado fue en la mayoría de los casos verdaderamente positivo, apreciándose un incremento importante 
en la calidad de las respuestas. Sí que debemos señalar que este aumento fue mucho mayor en aquellas preguntas que no requerían un desarrollo o argumentación.

Asumiendo el alumnado como un todo, pudimos observar una mejora bastante notable en el nivel de las respuestas, como se recoge en la Figura 3. En esta gráfica el nivel de la respuesta se evalúa al igual que en los cuestionarios (de 0 a 3 , según los niveles establecidos), pero obteniendo resultados generales del avance de la clase (a través del nivel medio de las respuestas a las 6 cuestiones del cuestionario). Como vemos, en el primer cuestionario, los resultados generales oscilan entre el nivel 1 y el 2 , sin llegar a este en ningún caso. En el cuestionario final, todos los resultados son superiores al nivel 2, llegando casi al nivel 3 en su totalidad en la cuestión 3.

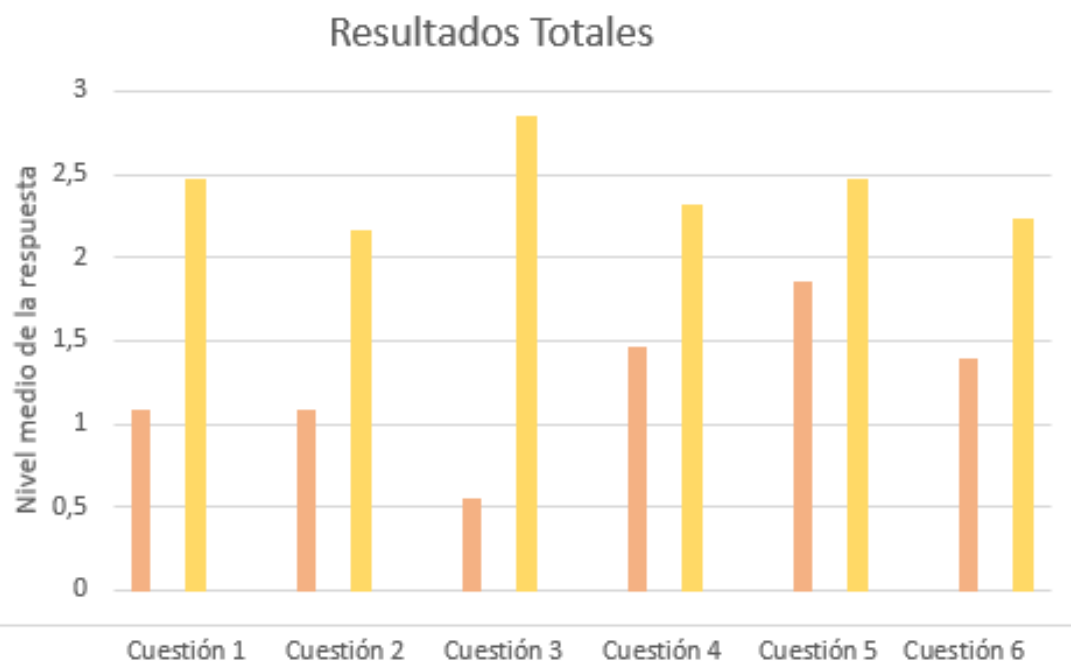

Figura 3. Gráfica comparativa del nivel medio de conocimiento del alumnado en los cuestiones de los cuestionarios inicial (rojo) y final (amarillo).

Jornadas de Formación e Innovación Docente del Profesorado | № 2 (2019) Esta obra se distribuye con la licencia Creative Commons Reconocimiento-NoComercial-SinObraDerivada 


\section{Conclusiones finales y evaluación del Ciclo de Mejora en el Aula}

Teniendo en consideración los resultados del ciclo de mejora, consideramos que, pese a que somos conscientes de su eficacia, existen ciertos aspectos a mejorar o modificar. Un ejemplo claro es la planificación de las actividades, quizás demasiado encorsetadas. En este caso para un nuevo ciclo de mejora plantearíamos actividades con un tiempo de duración más amplio o con mayor posibilidad de cambio, para así evitar quedarnos sin tiempo o que este en algunos casos sobre, es decir, buscaremos facilitar la adaptación de los tiempos de realización de las actividades a su momento.

Este ciclo de mejora nos ha permitido observar de una forma mucho más clara los beneficios de aplicar una metodología innovadora o simplemente diferente a las clases y docencia universitaria tradicional. La asimilación de este sistema permite que el alumnado sea mucho más participativo y demuestre más interés en los temas, ya que el sistema lo "obliga" a interactuar. Esto es beneficioso para su aprendizaje, pero también para la satisfacción personal y profesional del docente, cuya motivación e implicación, como sabemos, es fundamental para que este aprendizaje sea eficaz.

Los resultados positivos que pudimos valorar con los cuestionarios iniciales y finales son una motivación para aplicar este sistema en el resto de mis clases. Sin embargo, considero, que sería interesante realizar un estudio comparativo de los cuestionarios iniciales y finales comparando dos casos de estudio en los que se apliquen diferentes metodologías docentes, para evaluar la eficacia de los diferentes métodos de manera empírica y comparada.

La aplicación de este ciclo ha supuesto mucho más trabajo y tiempo en cuanto a su diseño, planteamiento y 
sobre todo desarrollo. Aunque en un primer momento se planteara como unas únicas seis horas, es imposible abordar tantas actividades en tan poco tiempo. El planear la asignatura es sin duda otro gran reto, ya que organizar tantas horas, sesiones y actividades puede ser una ardua labor. Sin embargo, si tenemos en cuenta que esto puede ser movible, podemos planear y diseñar bloques temáticos mucho más sintéticos y con el contenido que realmente queremos y consideramos relevante y necesario.

Finalmente, considero necesario señalar que la eficacia de este modelo didáctico depende de manera totalmente directa de las "preguntas clave". Formular preguntas verdaderamente interesantes y de interés puede ser una tarea dificil, ya que depende, y varía enormemente, de la temática a abordar y por supuesto de la creatividad o las ideas del docente. Consideramos esto extremadamente útil en la práctica docente, por lo que será algo que utilizaremos desde ahora en nuestras clases en la medida de lo posible.

Jornadas de Formación e Innovación Docente del Profesorado | № 2 (2019) Esta obra se distribuye con la licencia Creative Commons 


\section{Referencias bibliográficas}

Bain, K. (2006). Lo que hacen los mejores profesores universitarios. Traducción de Oscar Barberá, Valencia: Publicacions de la Universitat de València.

Cowan, N. (2001): The magical number 4 in short-term memory: A reconsideration of mental storage capacity. Behavioral and Brain Sciences, 24(1), 87-114.

De Alba, N. y Porlán, R. (2017). La metodología de enseñanza. En R. Porlán (Coord.), Enseñanza Universitarla. Cómo mejorarla (pp. 37-54). Madrid: Morata.

Finkel, D. (2008). Dar clase con la boca cerrada. Traducción de Óscar Barberá. Valencia: Publicacions de la Universitat de València (1a ed. inglesa, 2000).

García Díaz, E., Porlán, R. y Navarro, E. (2017). Los fines y los contenidos de enseñanza. En R. Porlán (Coord.) (2017). Enseñanza Universitaria. Cómo mejorarla (pp. 56-72). Madrid: Ediciones Morata.

García Pérez, F. F. (2000): Los modelos didácticos como instrumento de análisis y de intervención en la realidad educativa. Biblio 3W. Revista Bibliográfica de Geografia y Ciencias Sociales, V(207), 18 de febrero de 2000. Recuperado de http://www.ub.edu/geocrit/b3w-207.htm

Guerra Martín, M. D., Lima, M. y Lima, J. S. (2017). Effectiveness of Tutoring to Improve Academic Performance in Nursing Students at the University of Seville. Journal of New Approaches in Educational Research, 6(2), 93-102.

Gureckis, T. M. y Markant, D. B. (2012). Self-Directed Learning: a Cognitive and Computational Perspective. Perspectives on Psychological Science, 7(5), 464-481.

Rivero, A. y Porlán R. (2017). La evaluación en la enseñanza universitaria. En R. Porlán (Coord.), Enseñanza Universitaria. Cómo mejorarla (pp. 73- 91). Madrid: Morata. 